Article

\title{
Genetic Variation Putatively Associated with Mycobacterium tuberculosis Resistance to Perchlozone, a New Thiosemicarbazone: Clues from Whole Genome Sequencing and Implications for Treatment of Multidrug-Resistant Tuberculosis
}

\author{
Igor Mokrousov ${ }^{1, *}$, Anna Vyazovaya ${ }^{1}$, Gulnora Akhmedova ${ }^{2}$, Natalia Solovieva ${ }^{3}$, \\ Eugeni Turkin ${ }^{2}$ and Viacheslav Zhuravlev ${ }^{3}$ \\ 1 Laboratory of Molecular Epidemiology and Evolutionary Genetics, St. Petersburg Pasteur Institute, \\ 197101 St. Petersburg, Russia; elmtree2001@mail.ru \\ 2 Kaliningrad Regional Anti-Tuberculosis Dispensary, 236010 Kaliningrad, Russia; \\ gmakhmedova@mail.ru (G.A.); pt-disp@infomed39.ru (E.T.) \\ 3 St. Petersburg Research Institute of Phthisiopulmonology, 197101 St. Petersburg, Russia; \\ baclab@spbniif.ru (N.S.); jouravlev-slava@mail.ru (V.Z.) \\ * Correspondence: imokrousov@mail.ru
}

Received: 7 September 2020; Accepted: 2 October 2020; Published: 3 October 2020

\begin{abstract}
Perchlozone ([PCZ] 4-thioureido-iminomethylpyridinium perchlorate) is a new thiosemicarbazone approved for the treatment of multidrug-resistant tuberculosis (MDR-TB) in Russia and some other countries. The eth $A$ and hadABC mutations may confer PCZ resistance. At the same time, eth $A$ mutations are known to mediate resistance to ethionamide $(\mathrm{ETH})$ and prothionamide $(\mathrm{PTH})$. We aimed to study the genetic variation underlying Mycobacterium tuberculosis resistance to PCZ through whole genome sequencing (WGS) of consecutive isolates recovered during long-term treatment. This prospective study included patients admitted in 2018-2019 to the regional tuberculosis dispensary, Kaliningrad, Russia, whose treatment regimen included PCZ. Multiple M. tuberculosis isolates were recovered during PCZ treatment, and the bacterial DNA was subjected to WGS followed by bioinformatics analysis. We identified mutations in the genes putatively associated with PCZ resistance, eth $A$, and hadA. The most frequent one was a frameshift eth $A 106 \mathrm{GA}>\mathrm{G}$ (seven of nine patients) and most of the other mutations were also likely present before PCZ treatment. In one patient, a frameshift mutation eth $A 702 \mathrm{CT}>\mathrm{C}$ emerged after six months of PCZ treatment. A frequent presence of cross-resistance mutations to PCZ and ETH/PTH should be taken into consideration when $\mathrm{PCZ}$ is included in the treatment regimen of MDR-TB patients.
\end{abstract}

Keywords: Mycobacterium tuberculosis; drug resistance; perchlozone; thiosemicarbazone; ethionamide; ethA; had $A B C$

\section{Introduction}

Emergence and spread of multidrug or extensively drug-resistant (MDR/XDR) Mycobacterium tuberculosis strains highlight the importance of the development of new anti-tuberculosis drugs. However, long-term chemotherapy regimens involving such drugs are challenging because they are: (i) expensive, (ii) susceptible to noncompliance, (iii) adverse for a patient, due to side effects, and (iv) favorable to the pathogen, due to the extended time of adaptation. Perchlozone ${ }^{\circledR}$ (4-thioureido-iminomethylpyridinium perchlorate [PCZ]) is a new thiosemicarbazone approved in Russia, along with bedaquiline and delamanid, for treatment of MDR/XDR tuberculosis (TB) [1-4]. 
The drug was also approved for use or is in the process of registration in some other countries (https://patentscope.wipo.int/search/en/detail.jsf?docId=WO2011132114\&tab=NATIONALPHASE). PCZ is similar to thiacetazone (TAC) and differs from it by the side chain attached to the thiosemicarbazone moiety. Thiacetazone (TAC) was formerly used in combination with isoniazid to treat patients infected with MDR M. tuberculosis strains but was removed from the antitubercular chemotherapy due to its secondary toxic effects. TAC is a prodrug that is activated by the flavin-containing monooxygenase EthA to exert its antimycobacterial activity, and mutations in eth $A$ are associated with TAC resistance in $M$. tuberculosis [5]. Upon activation, TAC binds to the HadA component of the HadABC dehydratase complex, leading to inhibition of mycolic acid biosynthesis [6,7]. Similarly, PCZ is a prodrug that is activated by EthA and inhibits the HadABC complex. A cross-resistance to TAC and PCZ was shown by in vitro experiments and was mediated by both eth $A$ and hadA mutations [8]. On the other hand, hadA mutations may be acquired by $M$. tuberculosis as a resistance mechanism not only to TAC but also to isoxyl and flavonoids [9]. EthA is known to activate second-line drugs ethionamide (ETH) and prothionamide (PTH), whereas ethA or ethR mutations were described as one of the ETH/PTH resistance mechanisms [10,11].

In this study, we aimed to gain insight into the molecular basis of PCZ resistance including dynamic changes in M. tuberculosis genome during long-term treatment. To this end, we applied next-generation, whole-genome sequencing to the isolates consecutively recovered from patients who received PCZ as part of their chemotherapy regimen.

\section{Results}

In total, 35 isolates were recovered from nine patients who received PCZ (2-6 isolates from each, median four isolates per patient). The first available isolates were resistant to 6 to 11 drugs, including ETH or PTH (Table 1). One patient had MDR-TB, one had pre-XDR-TB and seven had XDR-TB. In addition, two $\mathrm{XDR}-\mathrm{TB}$ patients (three and five consecutive isolates) who received treatment with new drugs but not PCZ, were included in this study (Table 2).

Table 1. Summary of enrolled tuberculosis patients and infecting M. tuberculosis isolates for patients treated with perchlozone.

\begin{tabular}{|c|c|c|c|c|}
\hline Pt. & $\begin{array}{l}\text { Phenotypic Resistance } \\
\text { Profile of the First Isolate }\end{array}$ & Treatment Scheme & $\begin{array}{l}\text { Possible PCZ Resistance Mutations: } \\
\text { Position in the Reference Genome, } \\
\text { Position in Gene, Comment }\end{array}$ & $\begin{array}{l}\text { M. tuberculosis } \\
\text { Genotype }\end{array}$ \\
\hline 2. & HRSEKmCmEthOfxCsAmPAS & ZMfxLzdPczBq & $4327363 \mathrm{CT}>\mathrm{C}$, ethA $106 \mathrm{GA}>\mathrm{G}$, pre-existed & Beijing B0/W148 \\
\hline 3. & HRSEOfxEthPAS & CmZMfxTrdPczBq & $4327363 \mathrm{CT}>\mathrm{C}$, ethA $106 \mathrm{GA}>\mathrm{G}$, pre-existed & Beijing B0/W148 \\
\hline 4. & HSREKmCmEthOfxAmPAS & ZCsCmMfxLfxPczBq & $\begin{array}{c}4327363 \mathrm{CT}>\mathrm{C} \text {, eth } A 106 \mathrm{GA}>\mathrm{G} \text {, pre-existed } \\
4326770 \mathrm{TA}>\mathrm{T} \text {, eth } A 702 \mathrm{CT}>\mathrm{C} \text {, emerged }\end{array}$ & Beijing B0/W148 \\
\hline 7. & HRSEKmCmAmPthOfxPASCs & ZLfxTrdPczBq & $\begin{array}{c}4326533 \mathrm{G}>\mathrm{A} \text {, ethA } 314 \mathrm{ACC}>\mathrm{ATC} / \mathrm{Thr}> \\
\text { Ile, likely pre-existed }\end{array}$ & Beijing CAO \\
\hline 8. & HRSZKmCmAmOfxPth & ECsLfxLzdPczBq & $\begin{array}{c}4326533 \mathrm{G}>\mathrm{A}, \text { ethA } 314 \mathrm{ACC}>\mathrm{ATC} / \mathrm{Thr}> \\
\text { Ile, likely pre-existed }\end{array}$ & Beijing CAO \\
\hline 9. & HSREKmEthOfxAmZPASCs & ZLfxClzPczBq & $\begin{array}{c}4327363 \text { CT > C, ethA } 106 \text { GA > G, pre-existed } \\
731967 \text { G > C, hadA } 13 \text { CGG > CCC/Arg > } \\
\text { Pro, pre-existed }\end{array}$ & Beijing B0/W148 \\
\hline
\end{tabular}

Abbreviations. FCT: fibrocavernous TB, pulmonary TB: PTB. CAO: Central Asia outbreak. Antibiotics: $\mathrm{H}$-isoniazid, R-rifampin, S-streptomycin, E-ethambutol, Lfx-levofloxacin, Mfx-moxifloxacin, Ofx-ofloxacin, Cs-cycloserine, Am-amikacin, $\mathrm{Km}$-kanamycin, $\mathrm{Cm}$-capreomycin, PAS-Para-aminosalicylic acid, $\mathrm{Z}$-pyrazinamide, Pcz-perchlozone, Bq-bedaquiline, Clz-clofazimine, Lz-linezolid, Trd-Terizidone, Eth-ethionamide, Pth—prothionamide.

Based on the phylogenetically robust markers (single nucleotide polymorphisms (SNP) and deletions), isolates from all patients were assigned to the East-Asian lineage and the Beijing genotype (Table 1; Table 2). The isolates were further subdivided into Beijing B0/W148-cluster (nine patients) and Beijing Central Asia outbreak (CAO) clade (two patients). 
Table 2. Summary of enrolled tuberculosis patients and infecting M. tuberculosis isolates for patients not treated with perchlozone.

\begin{tabular}{ccccc}
\hline Pt & $\begin{array}{c}\text { Phenotypic Resistance } \\
\text { Profile of the First Isolate }\end{array}$ & Treatment Scheme & $\begin{array}{c}\text { Possible PCZ Resistance Mutations: } \\
\text { Position in the Reference Genome, } \\
\text { Position in Gene, Comment }\end{array}$ & $\begin{array}{c}\text { M. tuberculosis } \\
\text { Genotype }\end{array}$ \\
\hline 6. & HRSEKmOfx & ZCmMfxTrdPthBq & 4327363 CT $>$ C, ethA 106 GA $>$ G, pre-existed & Beijing B0/W148 \\
10. & HSREKmEtoOfxCs & AmZLfxPASBq & - & Beijing B0/W148 \\
\hline
\end{tabular}

Abbreviations. FCT: fibrocavernous TB, pulmonary TB: PTB. CAO: Central Asia outbreak. Antibiotics: $\mathrm{H}$-isoniazid, R-rifampin, S-streptomycin, E-ethambutol, Lfx-levofloxacin, Mfx-moxifloxacin, Ofx-ofloxacin, Cs-cycloserine, Am-amikacin, Km-kanamycin, Cm-capreomycin, PAS-Para-aminosalicylic acid, Z-pyrazinamide, Pcz-perchlozone, Bq-bedaquiline, Clz-clofazimine, Lz-linezolid, Trd-Terizidone, Eth-ethionamide, Pth-prothionamide.

The first isolates from nine patients infected with Beijing B0/W148 strain differed in $\geq 25$ SNPs and the first isolates from two patients infected with Beijing CAO strain differed in 29 SNPs (PE and PPE genes, and intergenic regions were excluded from analysis). Accordingly, we conclude that the analyzed strains were not directly epidemiologically linked and did not represent chains of recent transmission.

\subsection{Mutations: Frequency and Impact}

Analysis of genes associated with PCZ resistance (ethA, ethR, and hadABC) identified four mutations: three in eth $A$ and one in hadA (Table 1; Figure 1). No mutations in the promoter regions of the two genes were detected. The most frequent $e t h A$ mutation was found for seven of nine PCZ-treated patients and was a frameshift at gene position $106 \mathrm{GA}>\mathrm{G}$ (genome position 4,327,363 CT >C). This results in the premature stop codon 62-TAG and considerably abridged 61 amino acid protein.
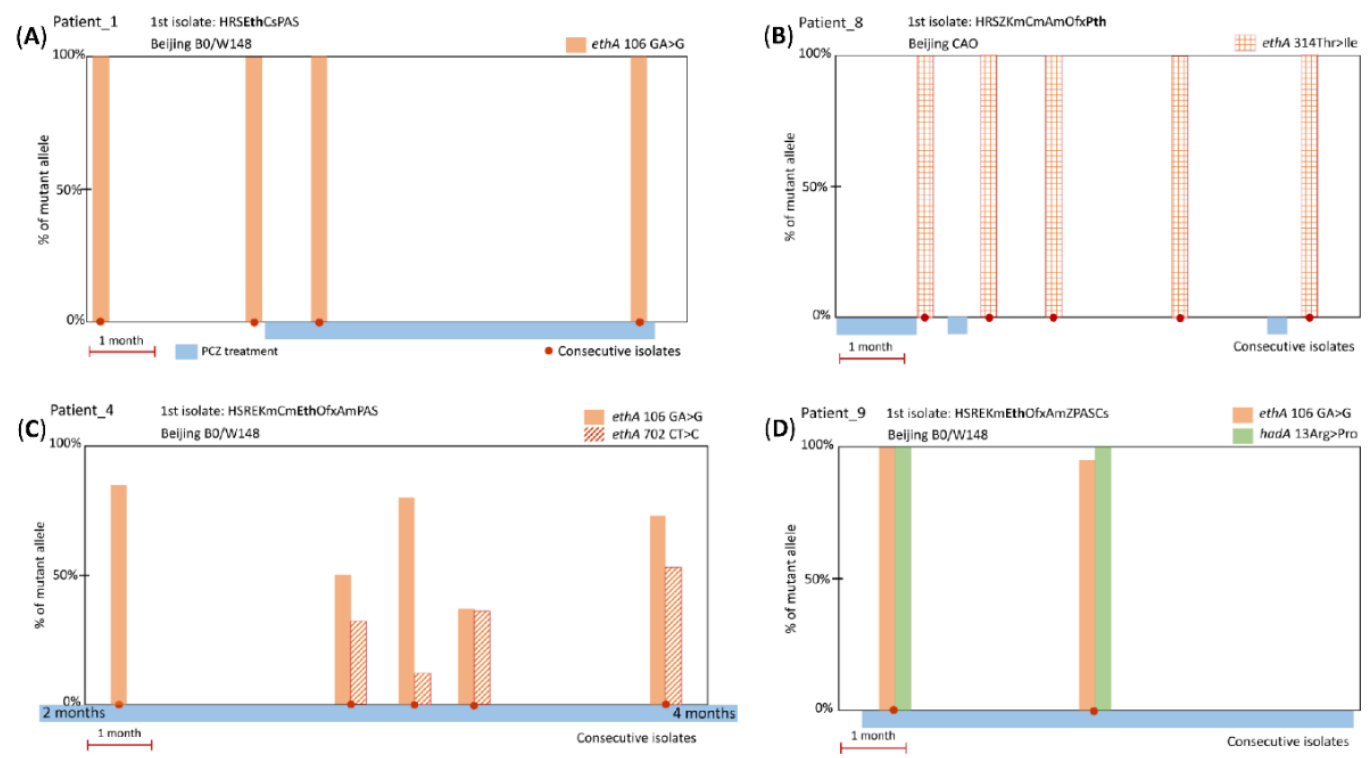

Figure 1. Representative examples of timelines of PCZ treatment, and ethA or hadA mutations in $M$. tuberculosis isolates. (A) Frameshift ethA $106 \mathrm{GA}>\mathrm{G}$ mutation present in PCZ pre-treatment isolates (5 patients); (B) substitution ethA 314 ACC > ATC/Thr > Ile (2 patients); (C) frameshift ethA 702 CT > $\mathrm{C}$ emerged during long-term PCZ treatment, in addition to the likely pre-existing ethA $106 \mathrm{GA}>\mathrm{G}$ (1 patient); (D) substitution hadA 13 CGG > CCC/Arg > Pro and frameshift ethA 106 GA > G (1 patient).

The other three mutations were found in one or two cases and two of them were accompanied by the aforementioned mutation eth $106 \mathrm{GA}>\mathrm{G}$ (Figure 1). The eth $A$ mutation at gene position $702 \mathrm{CT}>\mathrm{C}$ (genome position 4,326,770 TA $>\mathrm{T}$ ) is located inside codon 235 leading to premature stop codon 265-TGA and half-abridged 264 amino acid protein; it was found in one case and was present along with predominant ethA $106 \mathrm{GA}>\mathrm{G}$ mutation (Figure 1C). 
Two other mutations result in amino acid substitutions: (i) ethA 314 ACC $>$ ATC in two cases (Figure 1B) and (ii) hadA 13 CGG > CCC in one case (Figure 1D). Both these amino acid changes had low PAM1 scores (Table 3) implying their low probability, which may be interpreted as evidence that these mutations are beneficial to the mycobacterial survival since they were selected and retained.

Table 3. Mutations in genes putatively associated with PCZ resistance in M. tuberculosis isolates recovered from PCZ-treated patients.

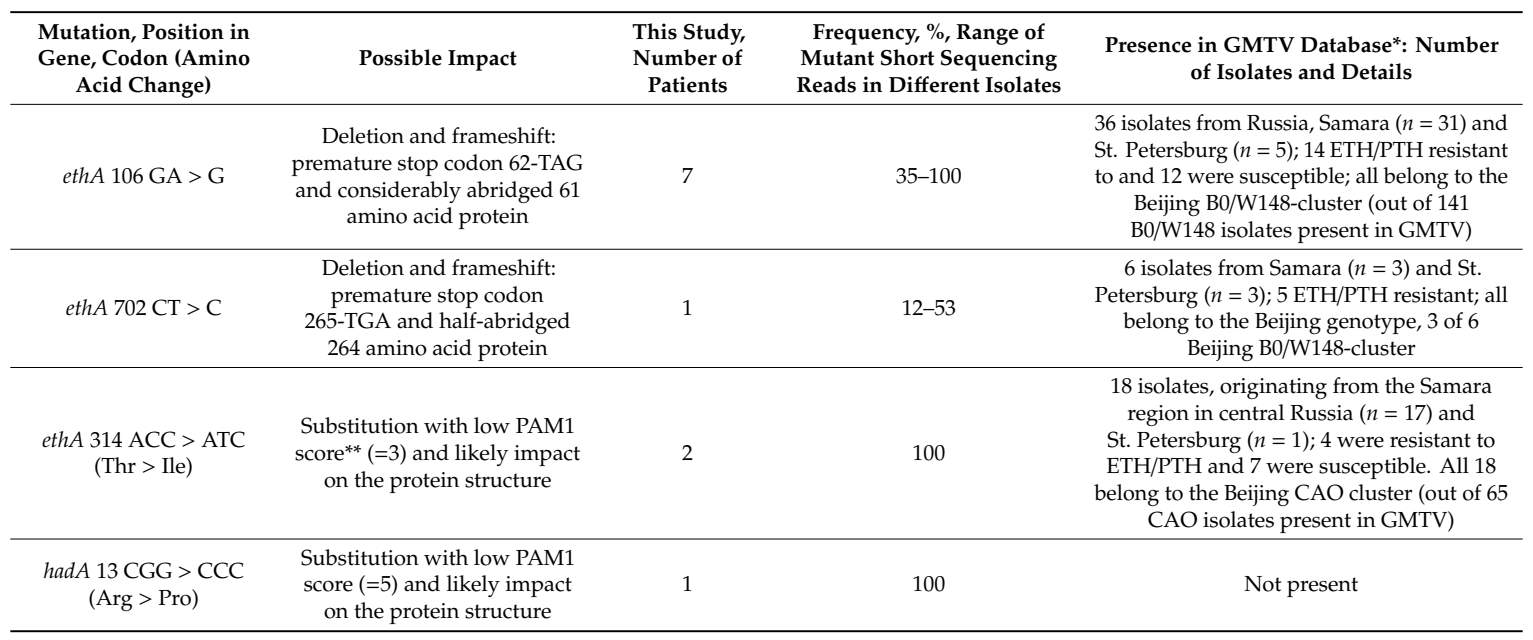

* Genome-based Mycobacterium tuberculosis Variation Database (GMTV): https://mtb.dobzhanskycenter.org/cgi-bin/ beta/main.py\#custom/world. ** PAM1—Point Accepted Mutation 1; it gives the probability (multiplied with 10,000) for the particular amino acid exchange to occur, given that $1 \%$ of the amino acids are changed.

The ethA $106 \mathrm{GA}>\mathrm{G}$ mutation was found in the isolates from one of two patients who did not receive PCZ treatment. It was present in all isolates from this patient in $100 \%$ reads and all isolates were ETH-resistant (Table 2).

\subsection{Mutations: Dynamic Changes}

Analysis of the consecutive isolates could be helpful in observing gradual development or fluctuation of resistance in response to the drug selective pressure. However, in most cases, all isolates had $100 \%$ mutant reads and this may be considered as additional evidence of the previous selection of these mutations during long-term ETH/PTH treatment. In few cases with initial 100\% ethA frameshift mutation, a wild type allele seemingly reappeared at 5-10\% in subsequent isolates (see ethA 106 GA > G in Figure 1D). Since a reversion to wild type is improbable, this could be explained by the within-patient heterogeneity of the M. tuberculosis strain population and different depth of WGS in different experiments.

A genuine change in the prevalence of mutant and wild type alleles was observed only in one patient. As we hypothesized above, the second mutation ethA $702 \mathrm{CT}>\mathrm{C}$ emerged after six months of PCZ treatment at 35\% frequency and its prevalence in the subsequent isolates fluctuated from $12 \%$ to $53 \%$ (Figure 1C). These two frameshift ethA mutations $106 \mathrm{GA}>\mathrm{G}$ and $702 \mathrm{CT}>\mathrm{C}$ are separated by $\sim 600 \mathrm{bp}$ and it is not possible to conclude from the WGS data whether they cosegregated on the same $\mathrm{read} /$ clone or not.

\section{Discussion}

A decision to include any additional drug into the complex treatment regimen of MDR/XDR-TB patients should be based on clinical recommendations and informed understanding of possible risks arising, in particular, from cross-resistance to other drugs. To date, only one report-an in vitro study-analyzed the emergence of PCZ resistance [8], and knowledge of the molecular mechanisms of M. tuberculosis resistance to this purportedly efficacious drug $[3,12]$ is very limited. Such knowledge 
is necessary and crucial given that PCZ is included in the list of essential drugs recommended for multidrug-resistant tuberculosis (MDR-TB) chemotherapy by the National Clinical Guidelines endorsed by the National Association of Phthisiatrists in Russia [13]. In turn, the impact of the Russian pool of M. tuberculosis strains on the global situation with MDR-TB is undeniable.

A high diversity of mutations scattered throughout the ethA gene was identified in the previous studies of the ETH/PTH resistance, where different kinds of mutations were described, including indels or nonsense mutations leading to premature stop codons [11,14,15]. If the EthA activity is lost, ETH is not converted to its active form, which explains the emergence of drug resistance. The lack of dominant ethA mutations in ETH-resistant isolates was speculatively attributed to the presence of monooxygenase homologs in $M$. tuberculosis that could protect the cells in case of inactive EthA [16,17]. At the same time, premature stop codons in eth $A$ also occurred in susceptible strains and at least two different explanations were proposed, e.g., existence of another ETH-activating enzyme, or a selective pressure by ETH that favors premature stop codon in ethA leading to low-levels of resistance [18].

In this study, the first isolates from all nine patients who received PCZ treatment were ETH- or PTH-resistant and the ethA mutations were present prior to PCZ treatment in most cases. These mutations could be either acquired during earlier ETH/PTH treatment or a patient could be infected with a resistant strain. The ethA $106 \mathrm{GA}>\mathrm{G}$ frameshift mutation was present in $100 \%$ reads in all pre-PCZ treatment isolates and could be acquired as a mechanism of ETH/PTH resistance (Figure 1A). The substitution ethA Thr314Ile was present in 100\% reads of the first isolate after 32 days of treatment (Figure 1B) but this timeframe seems too rapid for a mutation not only to emerge but to reach such total dominance in the population. A pre-treatment isolate was not available but it seems more likely that this mutation was present in the infecting strain before PCZ treatment. Only in one patient, the second ethA mutation apparently emerged after six months of PCZ treatment (Figure 1C). The observed fluctuation of the presence and prevalence of the frameshift mutations during treatment may speculatively correlate with either heteropopulation (since reversion is improbable) or the existence of one or more enzymes with functional redundancy to EthA.

Concerning hadA mutations, TAC and isoxyl are not used in Russia, and it is not known whether patient nine received these drugs (Figure 1D). However, 15 days of PCZ treatment is too short for a mutation not only to emerge but reach $100 \%$ frequency and the had $A$ mutation most likely existed before PCZ treatment.

In view of the presence of the particular ethA $106 \mathrm{GA}>\mathrm{G}$ frameshift mutation in pre-treatment isolates from six patients who did not receive previous PCZ treatment but were all phenotypically resistant to PTH or ETH, we assumed that this mutation emerged as PTH/ETH resistance mutation. We expanded this assumption to three other cases with this mutation. Thus, we conclude that the first isolates from seven of nine patients had this mutation before the PCZ treatment. Two mutations (substitutions ethA 314 ACC > ATC/Thr > Ile and hadA 13 CGG > CCC/Arg > Pro) were also likely present in isolates before PCZ treatment. Thus, only in one case, a mutation ethA $702 \mathrm{CT}>\mathrm{C}$ emerged after six months of the PCZ treatment.

We additionally looked for these mutations in the Genome-based Mycobacterium tuberculosis Variation (GMTV) database (https://mtb.dobzhanskycenter.org/cgi-bin/beta/main.py\# custom/world) [19], defined as a database integrating clinical, epidemiological, and microbiological description with genome variations based on whole genome sequencing (WGS) data of M. tuberculosis. While genomic variation information is generated by the database from raw genome data, the accompanying information, such as phenotypic drug susceptibility testing (DST), is provided by original laboratories or studies and cannot be verified.

The hadA 13 CGG > CCC mutation was not present in the GMTV database. The most known published hadA mutation is in codon 61 [20] and to the best of our knowledge, the described mutation in hadA13 is novel. On the other hand, all three ethA mutations identified in this study were present in GMTV and two of them (314 ACC > ATC and 106 GA > G) were detected in both ETH/PTH-resistant and susceptible isolates (Table 3). 
The ethA 314 ACC > ATC was detected in 18 Beijing CAO isolates, from Samara in Central Russia $(n=17)$ and St. Petersburg in Northwestern Russia $(n=1)$. This mutation was shown to be specific for the particular subcluster within the CAO clade, which included isolates from Russia and Uzbekistan [21]. We hypothesize that this mutation was selected in the ancestral founding strain of this branch either in response to ETH treatment or as neutral or fitness mutation, in both cases being sufficiently beneficial for mycobacterial survival and transmission.

The ethA 106 GA > G was found in the GMTV database in 36 Russian isolates and all belonged to the Beijing B0/W148-cluster (out of 141 B0/W148-cluster isolates present in GMTV).

In contrast to the above two eth $A$ mutations detected in both ETH/PTH-resistant and susceptible isolates, the ethA $702 \mathrm{CT}>\mathrm{C}$ was present in GMTV in six Russian isolates of the Beijing genotype and all five with available DST data were ETH/PTH-resistant. Only three of these six isolates belonged to the Beijing B0/W148 cluster and it may be that this mutation was a homoplastic change independently emerged in unrelated isolates and causatively linked to the ETH/PTH resistance. Otherwise, the information on phenotypic resistance to ETH/PTH should be regarded with caution since DST for these two drugs is known to be problematic and analysis of $\operatorname{inh} A$, ethA, and ethR is recommended as a reference method by WHO [22]. This situation highlights a controversy between the above-cited articles (where DST is considered a gold standard) and WHO guidelines as to what approach to the ETH resistance testing should be adopted as the primary one.

In conclusion, the frequent presence of cross-resistance mutations to both PCZ and ETH/PTH presents an especially worrisome finding of this study (although on a positive side one may note that the emergence of PCZ resistance during PCZ treatment was rare). This situation raises a major concern with regard to the non-efficiency of PCZ in the treatment of a significant number of MDR-TB cases whose isolates may be additionally resistant to ETH/PTH. In view of the high and increasing burden of MDR-TB in Russia, ETH and PTH are frequently used to treat such patients. The ETH/PTH regimen can take at least 18-24 months as recommended by national and international guidelines [13,22] and ETH/PTH resistance can emerge quite frequently in clinical isolates, especially in MDR isolates [23].

The treatment regimen of the XDR-TB patients includes at least six effective drugs and the presence of PCZ resistance mutations may not be necessarily associated with treatment failure. However, the inclusion of the non-effective additional drug in the treatment regimen is impractical and may be adverse for a patient's health. To adequately assess the association of the identified mutations with PCZ resistance, an implementation of the phenotypic PCZ susceptibility testing is urgently needed. A large prospective study of the diverse $M$. tuberculosis collection is warranted to formulate the recommendations for optimal use of PCZ, taking into consideration possible ETH/PTH resistance of the isolates.

\section{Materials and Methods}

The study was performed in the Kaliningrad region of Russia. The TB incidence in Kaliningrad decreased from 134/100,000 in 2006 to 50.6/100,000 in 2015. Nevertheless, this latter figure exceeds both a mean figure for Northwest Russia $(40.7 / 100,000)$ and the country as a whole $(57.7 / 100,000)$. The rate of primary MDR-TB in the region increased from 23.9\% in 2010 to 30.5\% in 2015 (http://mednet.ru/). This region is located in the westernmost part of the Russian Federation and makes an exclave on the Baltic Sea inside the European Union separated from the mainland Russia by Lithuania and Poland. Virtually closed from foreign contact from the 1950s until 1991, Kaliningrad today is a busy Russian-European crossroad that has an impact on the epidemiological situation of communicable diseases not only in Russia but also in northern Europe [24].

This prospective study included 11 patients admitted to the regional tuberculosis dispensary of the Kaliningrad region in 2018-2019. Nine patients received PCZ as a part of chemotherapy. No links between patients were identified based on the standard epidemiological investigation. All samples used in this study were coded and lacked personal information about the patients, especially names and addresses, to maintain their anonymity. Patient-related data were obtained anonymously, and no 
individual patient information is disclosed in this article. The study was approved by the Ethics Committee of the Research Institute of Phthisiopulmonology (protocol 31.2 of February 27, 2017).

M. tuberculosis isolates were recovered at the 1-5 month intervals. M. tuberculosis DST for first- and second-line drugs was performed using a modified proportion method on the Middlebrook 7H9 liquid culture and Bactec MGIT 960 system (Becton Dickinson, Sparks, Md.) according to the manufacturer's instructions and national guidelines (order \#951 of 29 December 2014 "On the approval of guidelines for improving the diagnosis and treatment of respiratory tuberculosis", Ministry Healthcare of the Russian Federation). The critical drug concentrations used were $1.0 \mu \mathrm{g} / \mathrm{mL}$ for streptomycin, $0.1 \mu \mathrm{g} / \mathrm{mL}$ for isoniazid, $5.0 \mu \mathrm{g} / \mathrm{mL}$ for ethambutol, $1.0 \mu \mathrm{g} / \mathrm{mL}$ for rifampicin, $100 \mu \mathrm{g} / \mathrm{mL}$ for pyrazinamide, $1.0 \mu \mathrm{g} / \mathrm{mL}$ for amikacin, $2.5 \mu \mathrm{g} / \mathrm{mL}$ for capreomycin, $2.0 \mu \mathrm{g} / \mathrm{mL}$ for ofloxacin, $5 \mu \mathrm{g} / \mathrm{mL}$ for ethionamide [13]. The laboratory is externally quality assured by the System for External Quality Assessment "Center for External Quality Control of Clinical Laboratory Research" (Moscow, Russia).

A limitation of this study was that the phenotypic PCZ resistance data was not available for the studied isolates since no critical concentrations have been approved and PCZ susceptibility testing is not yet implemented. Based on the previous reports on PCZ and TAC resistance, we assumed mutations in ethA, ethR, and $h a A B C$ as a genetic proxy of phenotypic resistance to PCZ.

DNA was extracted from bacterial cultures grown on the Loewenstein-Jensen medium using a CTAB-based method as previously described [25].

Whole genome sequencing was performed at the MiSeq or NextSeq 500 platforms (Illumina). DNA libraries were prepared using ultrasound DNA fragmentation and NEBNext Ultra DNA Library Prep Kit for Illumina (New England Biolabs). Data for the M. tuberculosis sequenced genomes were deposited in the NCBI Sequence Read Archive (project numbers PRJNA525341 and PRJNA635788). The fastq files were aligned to the complete genome of the reference strain H37Rv (NC_00962.3) using Geneious 9 program (Biomatters, New Zealand) and additionally checked with PhyReSE online tool (https://bioinf.fz-borstel.de/mchips/phyresse/). The mean read length was $150 \mathrm{bp}$ for NextSeq 500 data and $250 \mathrm{bp}$ for MiSeq data. An average genome coverage for the analyzed genome regions was x103 for the NextSeq 500 data and x31 for the MiSeq data.

M. tuberculosis isolates (genomes) were assigned to the Beijing genotype and its specific subtypes based on the assessment of the previously described SNP markers and genomic deletions [26-30]. The short sequencing reads (fastq files) were subjected to in silico spoligotyping using TGS-TB online tool at https://gph.niid.go.jp/tgs-tb/ [31] to deduce their spoligoprofile.

Author Contributions: Conceptualization, I.M. and A.V.; investigation, G.A. and N.S.; writing-original draft preparation, I.M.; writing-review and editing, I.M., A.V., and V.Z.; supervision, I.M. and E.T.; project administration, A.V.; funding acquisition, A.V. All authors have read and agreed to the published version of the manuscript.

Funding: This research was funded by the Russian Science Foundation (grant 19-15-00028).

Conflicts of Interest: The authors declare no conflict of interest. The funders had no role in the design of the study; in the collection, analyses, or interpretation of data; in the writing of the manuscript, or in the decision to publish the results.

\section{References}

1. Vinogradova, T.I.; Aleksandrova, A.E.; Antonenkova, E.V.; Elokhina, V.N.; Nakhmanovich, A.S. Design and study of new agents having antitubercular activity: The original compound perchlosone as a potent agent of etiotropic therapy for tuberculosis. Probl. Tuberk. 1999, 3, 45-47. (In Russian)

2. Yablonskiy, P.K.; Vinogradova, T.I.; Levashev, Y.N.; Pavlova, M.V.; Zilber, E.K.; Starshinova, A.A.; Sapozhnikova, N.V.; Chernokhaeva, I.V.; Archakova, L.I.; Zabolotnykh, N.V.; et al. Preclinical and clinical trials of the new tuberculosis drug perchlozon. Ter Arkh. 2016, 88, 111-115. (In Russian) [CrossRef] [PubMed] 
3. Churilov, L.; Korzhikov-Vlakh, V.; Sinitsyna, E.; Polyakov, D.; Darashkevich, O.; Poida, M.; Platonova, G.; Vinogradova, T.; Utekhin, V.; Zabolotnykh, N.; et al. Enhanced delivery of 4-thioureidoiminomethyl pyridinium perchlorate in tuberculosis models with IgG functionalized poly(Lactic acid)-based particles. Pharmaceutics 2018, 11, 2. [CrossRef] [PubMed]

4. Gushchin, A.S.; Vinogradova, T.I.; Yablonskiy, P.K.; Batyunin, G.A.; Zabolotnyh, N.V.; Vasilieva, S.N.; Maligin, A.V. Tuberculosis Drug Based on 4-Thioureido-Iminomethylpyridinium Perchlorate: Method of Preparation and Treatment. U.S. Patent 9,750,727 B2, 5 September 2017. Available online: https: //patentscope.wipo.int/search/en/detail.jsf?docId=WO2011132114 (accessed on 27 September 2020).

5. Dover, L.G.; Alahari, A.; Gratraud, P.; Gomes, J.M.; Bhowruth, V.; Reynolds, R.C.; Besra, G.S.; Kremer, L. EthA, a common activator of thiocarbamide-containing drugs acting on different mycobacterial targets. Antimicrob. Agents Chemother. 2007, 51, 1055-1063. [CrossRef]

6. Halloum, I.; Viljoen, A.; Khanna, V.; Craig, D.; Bouchier, C.; Brosch, R.; Coxon, G.; Kremer, L. Resistance to thiacetazone derivatives active against Mycobacterium abscessus involves mutations in the MmpL5 transcriptional repressor MAB_4384. Antimicrob. Agents Chemother. 2017, 61, e02509-02516. [CrossRef]

7. Belardinelli, J.M.; Morbidoni, H.R. Mutations in the essential FAS II-hydroxyacylACP dehydratase complex confer resistance to thiacetazone in Mycobacterium tuberculosis and Mycobacterium kansasii. Mol. Microbiol. 2012, 86, 568-579. [CrossRef]

8. Gopal, P.; Dick, T. The new tuberculosis drug Perchlozone ${ }^{\circledR}$ shows cross-resistance with thiacetazone. Int. J. Antimicrob. Agents 2015, 45, 430-433. [CrossRef]

9. Dong, Y.; Qiu, X.; Shaw, N.; Xu, Y.; Sun, Y.; Li, X.; Li, J.; Rao, Z. Molecular basis for the inhibition of $\beta$-hydroxyacyl-ACP dehydratase HadAB complex from Mycobacterium tuberculosis by flavonoid inhibitors. Protein Cell. 2015, 6, 504-517. [CrossRef]

10. Islam, M.M.; Tan, Y.; Hameed, H.M.A.; Liu, Z.; Chhotaray, C.; Liu, Y.; Lu, Z.; Cai, X.; Tang, Y.; Gao, Y.; et al. Detection of novel mutations associated with independent resistance and cross-resistance to isoniazid and prothionamide in Mycobacterium tuberculosis clinical isolates. Clin. Microbiol. Infect. 2019, 25, 10041.e1-10041.e7. [CrossRef]

11. De Welzen, L.; Eldholm, V.; Maharaj, K.; Manson, A.L.; Earl, A.M.; Pym, A.M. Whole-transcriptome and -genome analysis of extensively drug-resistant Mycobacterium tuberculosis clinical Iisolates identifies downregulation of ethA as a mechanism of ethionamide resistance. Antimicrob. Agents Chemother. 2017, 61. [CrossRef]

12. Chernokhaeva, I.; Pavlova, M.; Starshinova, A.; Sapozhnikova, N.; Belaeva, E.; Zhuravlev, V.; Archakova, L.; Yablonskii, P.; Starshinova, A. Therapy of pulmonary tuberculosis with multidrug-resistant Mycobacterium tuberculosis using tioureidoiminomethylpyridinium perchlorate (Perchlozon). Int. J. Tech. Res. Appl. 2015, 3, 59-62.

13. Phthisiatry National Clinical Guidelines; Yablonsky, P.K. (Ed.) Moscow, Russia, 2015; p. 231, ISBN 978-5-97043675-2. Available online: https://spbniif.ru/upload/medialibrary/bd7/bd77ac5cda63ac2a20a0846242bb3db2. pdf (accessed on 27 September 2020).

14. Tan, Y.; Su, B.; Zheng, H.; Song, Y.; Wang, Y.; Pang, Y. Molecular characterization of prothionamide-resistant Mycobacterium tuberculosis isolates in Southern China. Front. Microbiol. 2017, 8, 2358. [CrossRef] [PubMed]

15. Rueda, J.; Realpe, T.; Mejia, G.I.; Zapata, E.; Rozo, J.C.; Ferro, B.E.; Robledo, J. Genotypic analysis of genes associated with independent resistance and cross-resistance to isoniazid and ethionamide in Mycobacterium tuberculosis clinical isolates. Antimicrob. Agents Chemother. 2015, 59, 7805-7810. [CrossRef] [PubMed]

16. Morlock, G.P.; Metchock, B.; Sikes, D.; Crawford, J.T.; Cooksey, R.C. ethA, inhA, and katG loci of ethionamide-resistant clinical Mycobacterium tuberculosis isolates. Antimicrob. Agents. Chemother. 2003, 47, 3799-3805. [CrossRef]

17. Vilchèze, C.; Jacobs, W.R. Resistance to isoniazid and ethionamide in Mycobacterium tuberculosis: Genes, mutations, and causalities. Microbiol. Spectr. 2014, 2, MGM2-MGM14. [CrossRef]

18. Johnsen, C.H.; Clausen, P.T.L.C.; Aarestrup, F.M.; Lund, O. Improved resistance prediction in Mycobacterium tuberculosis by better handling of insertions and deletions, premature stop codons, and filtering of non-informative sites. Front. Microbiol. 2019, 10, 2464. [CrossRef] 
19. Chernyaeva, E.N.; Shulgina, M.V.; Rotkevich, M.S.; Dobrynin, P.V.; Simonov, S.A.; Shitikov, E.A.; Ischenko, D.S.; Karpova, I.Y.; Kostryukova, E.S.; Ilina, E.N.; et al. Genome-wide Mycobacterium tuberculosis variation (GMTV) database: A new tool for integrating sequence variations and epidemiology. Bmc Genom. 2014, 15, 308. [CrossRef]

20. Grzegorzewicz, A.E.; Eynard, N.; Quémard, A.; North, E.J.; Margolis, A.; Lindenberger, J.J.; Jones, V.; Korduláková, J.; Brennan, P.J.; Lee, R.E.; et al. Covalent modification of the Mycobacterium tuberculosis FAS-II dehydratase by Isoxyl and Thiacetazone. ACS Infect. Dis. 2015, 1, 91-97. [CrossRef]

21. Köser, C. Implications of the Genetic Diversity within MTBC and M. canettii for the Development of New DST Assays. 2015. Available online: http://www.stoptb.org/wg/new_diagnostics/assets/documents/NDWD_ AnnMtg2015_02-03_Claudio_KOSER.pdf (accessed on 27 September 2020).

22. World Health Organization. WHO Consolidated Guidelines on Drug-Resistant Tuberculosis Treatment; World Health Organization: Geneva, Switzerland, 2019; Licence: CC BY-NC-SA 3.0 IGO.

23. Ismail, N.A.; Mvusi, L.; Nanoo, A.; Dreyer, A.; Omar, S.V.; Babatunde, S.; Molebatsi, T.; van der Walt, M.; Adelekan, A.; Deyde, V.; et al. Prevalence of drug-resistant tuberculosis and imputed burden in South Africa: A national and sub-national cross-sectional survey. Lancet Infect. Dis. 2018, 18, 779-787. [CrossRef]

24. Vyazovaya, A.A.; Akhmedova, G.M.; Solovieva, N.S.; Gerasimova, A.A.; Starkova, D.A.; Turkin, E.N.; Zhuravlev VYu Narvskaya, O.V.; Mokrousov, I.V. Molecular epidemiology of tuberculosis in the Kaliningrad region of Russia: 10 years after. Russ. J. Infect. Immun. 2017, 7, 367-374. (In Russian) [CrossRef]

25. van Embden, J.D.; Cave, M.D.; Crawford, J.T.; Dale, J.W.; Eisenach, K.D.; Gicquel, B.; Hermans, P.; Martin, C.; McAdam, R.; Shinnick, T.M.; et al. Strain identification of Mycobacterium tuberculosis by DNA fingerprinting: Recommendations for a standardized methodology. J. Clin. Microbiol. 1993, 31, 406-409. [CrossRef] [PubMed]

26. Tsolaki, A.G.; Gagneux, S.; Pym, A.S.; Goguet de la Salmoniere, Y.O.; Kreiswirth, B.N.; Van Soolingen, D.; Small, P.M. Genomic deletions classify the Beijing/W strains as a distinct genetic lineage of Mycobacterium tuberculosis. J. Clin. Microbiol. 2005, 43, 3185-3191. [CrossRef] [PubMed]

27. Mokrousov, I.; Narvskaya, O.; Vyazovaya, A.; Otten, T.; Jiao, W.W.; Gomes, L.L.; Suffys, P.N.; Shen, A.D.; Vishnevsky, B. Russian "successful” clone B0/W148 of Mycobacterium tuberculosis Beijing genotype: A multiplex PCR assay for rapid detection and global screening. J. Clin. Microbiol. 2012, 50, 3757-3759. [CrossRef] [PubMed]

28. Shitikov, E.; Kolchenko, S.; Mokrousov, I.; Bespyatykh, J.; Ischenko, D.; Ilina, E.; Govorun, V. Evolutionary pathway analysis and unified classification of East Asian lineage of Mycobacterium tuberculosis. Sci. Rep. 2017, 7, 9227. [CrossRef] [PubMed]

29. Mokrousov, I.; Chernyaeva, E.; Vyazovaya, A.; Skiba, Y.; Solovieva, N.; Valcheva, V.; Levina, K.; Malakhova, N.; Jiao, W.W.; Gomes, L.L.; et al. Rapid assay for detection of the epidemiologically important central Asian/Russian strain of the Mycobacterium tuberculosis Beijing genotype. J. Clin. Microbiol. 2018, 56, e01551-17. [CrossRef]

30. Shitikov, E.; Vyazovaya, A.; Malakhova, M.; Guliaev, A.; Bespyatykh, J.; Proshina, E.; Pasechnik, O.; Mokrousov, I. Simple assay for detection of the central Asia outbreak clade of the Mycobacterium tuberculosis Beijing genotype. J. Clin. Microbiol. 2019, 57, e00215-19. [CrossRef]

31. Sekizuka, T.; Yamashita, A.; Murase, Y.; Iwamoto, T.; Mitarai, S.; Kato, S.; Kuroda, M. TGS-TB: Total genotyping solution for Mycobacterium tuberculosis using short-read whole-genome sequencing. PLOS ONE 2015, 10, e0142951. [CrossRef]

(C) 2020 by the authors. Licensee MDPI, Basel, Switzerland. This article is an open access article distributed under the terms and conditions of the Creative Commons Attribution (CC BY) license (http://creativecommons.org/licenses/by/4.0/). 